\title{
Development of Computer Control Vibration Unit
}

\section{YAU Hon Keung*}

Department of System Engineering and Engineering Management, City University of Hong Kong, China

In our laboratory, we are equipped with a vibration unit to measure the human response under various vibration conditions. However, we found that it is not efficient as it is manually controlled; we need to upgrade it to a computer control vibration unit.

The vibration unit was enhanced using an extra hardware installation and the window-based software design control panel. The whole vibration unit contains 3 main parts: the frequency convertor, the driving motor and the vibrating platform. The frequency convertor receives signal from computer and give a specific current to the motor. The motor's shaft is connected to the vibrating platform by gears as the medium of transmitting power. With the rotation of the cam, the vibrating platform can accelerate/ decelerate in sine wave form up to the stated frequency. The vibration unit can operate at nine frequencies from $1.6 \mathrm{~Hz}$ to $10 \mathrm{~Hz}$ [1]. The vibrator can generate a sine wave form peak-to-peak stroke of $10 \mathrm{~mm}$. The vibration unit is designed to support up to $130 \mathrm{~kg}$ load, the vibrator's platform is connected to a $4 \mathrm{~kW}, 960$ $\mathrm{rpm}$ motor with the gear ratio of $1 / 1.54$. The motor chosen was a $4 \mathrm{~kW}$ with $960 \mathrm{rpm}$. A pair of gears with 1/1.54 gear ratio was connected to the motor shaft to reduce the angular speed and increase the resulting torque and power.

The block diagram of hardware of vibration unit is shown in Figure 1.

The BASIC Stamp module is a microcontroller developed by Parallax, Inc. It is easily programmed using a form of the BASIC programming language called PBASIC. It is called a "Stamp" simply because it is close to the size of an average postage stamp. Once the program is downloaded to the basic stamp, it can run independently without the connection of computer. The chosen basic stamp at this experiment is so called Basic Stamp II. The BASIC Stamp II can be run using a programmable microcontroller. It is able to control and monitor timers, keypads, motors, sensors, switches, relays, lights, etc. Basic

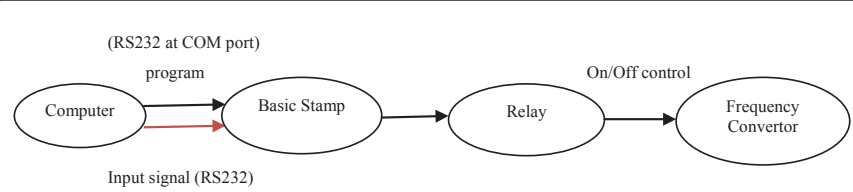

Figure 1: Hardware of vibration unit.

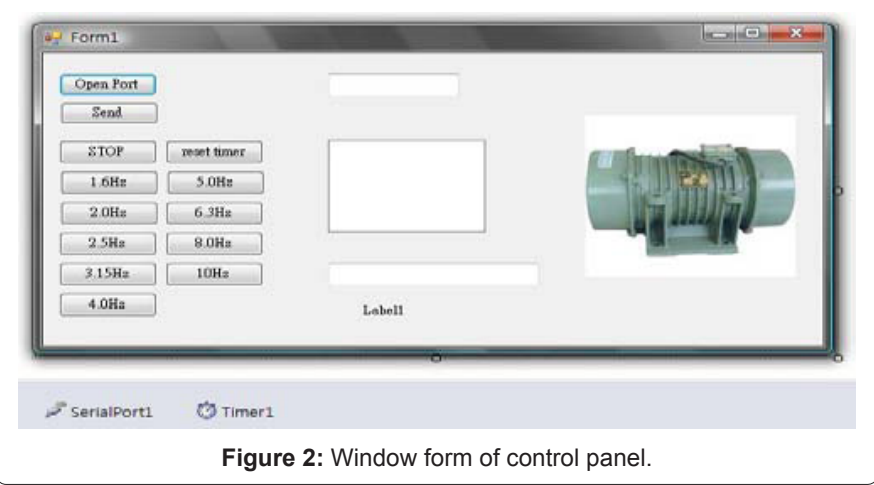

Stamp teaching board is designed and produced by the Parallax Inc for the purpose of control and signal transformation. This board is well developed and it can provide a small plug board for different purpose of trial for the component.

For the ease of control and user friendliness, we employ computer control of the vibration unit. A program is developed and this program also provides time counting function so that we can have the vibration and time counting at the same time. From the (Figure 2), we can see the outlook of the window form. While running the program, several steps are needed before we start the vibration unit.

1. Checking of the emergency stop button(function testing).

2. Ask the subject to sit on the platform "before" the vibration starts.

3. Press the open port before choosing the vibration frequency.

In conclusion, a computer control vibration unit was developed successfully. The vibration frequency of the unit can be easily controlled via the computer. The major limitation of the vibration unit is that only nine frequencies can be selected. The future research opportunity is that the vibration frequency can be adjusted randomly (instead of only 9 frequencies) using the computer.

\section{Reference}

1. Subashi GHMJ, Nawayseh N, Matsumoto Y, Griffin MJ (2009) Nonlinear subjective and dynamic responses of seated subjects exposed to horizontal whole-body vibration. J Sound Vib 321: 416-434

*Corresponding author: YAU Hon Keung, Instructor, Department of System Engineering and Engineering Management, City University of Hong Kong, China, E-mail: honkyau@cityu.edu.hk

Received July 26, 2012; Accepted July 28, 2012; Published July 31, 2012

Citation: YAU Hon Keung (2012) Development of Computer Control Vibration Unit. Adv Robot Autom 1:e109. doi:10.4172/2168-9695.1000e109

Copyright: @ 2012 YAU Hon Keung. This is an open-access article distributed under the terms of the Creative Commons Attribution License, which permits unrestricted use, distribution, and reproduction in any medium, provided the original author and source are credited. 